\title{
Germanica
}

\section{Autour de la BD germanophone : témoignages et perspectives}

\section{Frédéric Sauvage}

Traducteur : Ingeborg Rabenstein-Michel et Frédéric Sauvage

\section{(2) OpenEdition}

\section{Journals}

Édition électronique

URL : http://journals.openedition.org/germanica/1136

DOI : 10.4000/germanica.1136

ISSN : 2107-0784

Éditeur

Université de Lille

\section{Édition imprimée}

Date de publication : 31 décembre 2010

Pagination : 177-185

ISBN : 9782913857261

ISSN : 0984-2632

\section{Référence électronique}

Frédéric Sauvage, "Autour de la BD germanophone : témoignages et perspectives », Germanica [En ligne], 47 | 2010, mis en ligne le 01 décembre 2012, consulté le 06 octobre 2020. URL : http:// journals.openedition.org/germanica/1136 ; DOI : https://doi.org/10.4000/germanica.1136

Ce document a été généré automatiquement le 6 octobre 2020.

(c) Tous droits réservés 


\section{Autour de la BD germanophone : témoignages et perspectives}

\section{Frédéric Sauvage}

Traduction : Ingeborg Rabenstein-Michel et Frédéric Sauvage

1 Pour conclure ce volume, nous vous proposons de lire les témoignages de cinq personnalités du monde de la BD de langue allemande ${ }^{1}$. Trois dessinateurs (Isabel Kreitz, Nicolas Mahler, Barbara Yelin), une éditrice (Jutta Harms) et un scénariste (Peer Meter) ont répondu à sept questions concernant le passé, le présent et l'avenir de la bande dessinée dans les pays germanophones, laquelle se décline dorénavant de préférence sous forme de roman graphique («graphic novel»).

2 À souligner, l'arrivée massive des dessinatrices dans un univers jusque-là essentiellement masculin qu'elles contribuent de manière étonnante à faire évoluer.

3 Reconnu par la critique comme un art à part entière, la BD a dorénavant acquis ses lettres de noblesse et conquis un lectorat varié, qui n'hésite plus à l'inclure dans ses pratiques culturelles.

\section{Interviews}

Peut-on parler d'une nouvelle génération d'auteurs de BD germanophones, notamment dans le domaine du roman graphique (« graphic novel»), dont plusieurs ont été récemment traduits en français?

Barbara YELIN (BY) : Beaucoup de jeunes dessinateurs et dessinatrices ont en effet émergé au cours de ces dernières années. Sans doute, entre autres raisons, parce que des artistes enseignant à l'université (comme par exemple Anke Feuchtenberger, Atak ou encore Henning Wagenbreth) ont vigoureusement défendu ce genre. On remarque aussi, et c'est frappant, que la dimension narrative est passée au premier plan, après une période d'expérimentations plutôt visuelles. L'éventail est large - et le fait qu'un nombre grandissant de romans graphiques soit traduit en français témoigne aussi de l'intérêt du marché français. 
Nicolas MAHLER (NM) : Oui, aujourd'hui, bon nombre de dessinateurs livrent des histoires beaucoup plus longues, en comparaison avec la production d'il y a une quinzaine d'années. Le marché du livre a évolué, et les maisons d'édition s'ouvrent aussi tout doucement à la $\mathrm{BD}$, ce qui incite les dessinateurs à proposer à leur tour des histoires plus longues. Il y a quinze ans, on se heurtait à l'absence totale de possibilités de publication. Or, plus le nombre des publications est élevé, plus les jeunes dessinateurs se sentent motivés pour raconter leurs propres histoires.

La BD a, de plus, évolué du format classique (l'album) vers le format du livre. Cela a rendu possible la publication d'histoires plus personnelles. Avant, on ne trouvait des éditeurs que pour des BD d'aventure ou du genre fantastique, et qui étaient toujours en couleur.

Isabel KREITZ (IK) : Le roman graphique est perçu d'une manière très différente de la BD classique. On y aborde des thèmes plus littéraires qui intéressent aussi des lecteurs peu familiarisés avec le "comic ». Mais à mon avis, on devrait moins parler d'une nouvelle génération d'auteurs que d'une nouvelle génération d'éditeurs, avec de nouvelles stratégies de marketing qui nous permettent, à nous dessinateurs et auteurs, de publier aujourd'hui de manière très indépendante, et dans des formats qui intéressent aussi l'étranger.

Jutta HARMS (JH) : Oui : les jeunes auteurs privilégient les sujets captivants, le suspens et la narration. Leurs influences graphiques et visuelles viennent des auteurs qui ont radicalement renouvelé le langage visuel de la BD dans les années quatre-vingt dix, comme par exemple Anke Feuchtenberger, Martin tom Dieck, Atak. Et puis on constate que le marché français de la BD s'ouvre bien plus facilement qu'avant aux auteurs et dessinateurs étrangers.

Peer METER (PM) : Oui, absolument! Depuis que le « comic » a fait son entrée dans les écoles des Beaux-Arts en tant que discipline - Anke Feuchtenberger à Hambourg, Henning Wagenbreth à Berlin, Hendrik Dorgathen à Kassel, Martin tom Dieck à Essen, Atak à Halle-, l'université voit la BD d'un œil plus bienveillant. Deux pôles géographiques principaux existent aujourd'hui en Allemagne: Berlin et surtout Hambourg, où les cours d'Anke Feuchtenberger à l'École supérieure des arts plastiques ont fait émerger toute une génération de jeunes dessinateurs.

Il y a beaucoup de femmes parmi les auteurs à succès, ces dernières années : peut-on dire qu'elles apportent un nouveau souffle à la $\mathrm{BD}$, au roman graphique de langue allemande?

BY : En investissant de plus en plus le domaine de la BD, les femmes introduisent indubitablement des approches nouvelles. Je ne veux pas généraliser, mais je pense que les femmes peuvent apporter à ce medium des sujets et des éclairages nouveaux. J'ai constaté - mais ce n'est que ma propre expérience - que les femmes se sont familiarisées plus tardivement que leurs collègues masculins avec la BD - en général seulement pendant leurs études ou leur formation. C'est un avantage comme un inconvénient, mais on pourrait dire qu'elles abordent le «comic» avec des objectifs très différents, de manière plus décontractée. C'est une approche qui permet d'élargir les frontières du genre. La nouvelle génération manga fonctionne quant à elle de manière encore différente et inédite, et là aussi, on trouve de nombreuses jeunes dessinatrices.

NM : Oui, tout à fait. Pendant longtemps, les BD étaient suspectées d'être de simples histoires de héros et de garnements, et cela explique sans doute le peu d'intérêt pour ce métier que montraient les dessinatrices dans le passé. Mais depuis la parution de 
livres comme Persepolis, les BD sont perçues fort heureusement de manière un peu plus nuancée, elles portent moins automatiquement l'étiquette de "sous-littérature pour les garçons ». Et plus il y aura de publications de ce genre, plus les dessinatrices s'intéresseront aux histoires en images.

IK : Le mode de narration plus épique propre au roman graphique permet d'aborder des sujets autres que ceux de la BD classique, qui s'adressait plutôt à un public masculin. C'est peut-être la raison pour laquelle les auteures recourent plus qu'avant à cette forme d'expression. Et l'élargissement au public féminin qui en résulte augmente le chiffre de vente des "comics »... On observe une évolution analogue depuis pas mal de temps déjà dans le domaine des mangas.

$\mathrm{JH}$ : Les jeunes dessinatrices racontent évidemment des histoires très différentes de celles de leurs collègues masculins, ce qui ne veut cependant pas dire que leurs sujets seraient «typiquement féminins ». Aujourd'hui, on peut dire que les dessinateurs en général s'intéressent à des thèmes bien plus variés que ne l'étaient ceux des albums classiques, qui privilégiaient l'action, la fantaisie et l'humour.

PM : Absolument! C'est un vrai phénomène! Ce sont surtout les jeunes dessinatrices qui confèrent leur particularité à la BD allemande. Il n'y a pas si longtemps que ça, on considérait avec beaucoup de condescendance les travaux proposés par des femmes, qui étaient appelés les « comics pour les filles ». Aujourd'hui, tout ça a radicalement changé. Mais je ne saurais dire si ces dessinatrices traitent de sujets tout à fait nouveaux ; après tout, chaque dessinateur, chaque dessinatrice a des thèmes qui lui sont propres. Mais elles apportent très certainement une nouvelle sensibilité, et leur vision féminine de la BD est un enrichissement pour tous.

Est-ce que le développement des romans graphiques (existe-t-il une dénomination allemande?) marque un tournant en Allemagne? Ou doit-on y voir seulement un nouveau positionnement des éditeurs, notamment par rapport aux mangas?

BY : Eh bien, ce n'est après tout qu'une manière de désigner une BD qui se rapproche $\mathrm{du}$ roman. Il s'agit de permettre une identification facile et attractive pour les lecteurs adultes, qui, en voyant le terme de "comic», continuaient d'imaginer exclusivement des histoires drôles pour enfants. Qui auraient aimé trouver des histoires plus exigeantes, avec plus de contenu et plus de substance graphique, sans même savoir que cela pouvait exister. Tout cela est en train de changer, et c'est pour cette raison qu'un tel positionnement est extrêmement utile.

$\mathrm{NM}$ : Je ne connais pas assez les mangas pour pouvoir répondre à cette question. Je ne vois pas trop leur influence sur le travail de la génération de dessinateurs qui ont entre trente et quarante ans aujourd'hui. Je crois que cette influence sera plus perceptible pour la génération suivante. Les gens qui publient actuellement s'inspirent à mon avis plutôt de modèles français ou américains...

IK : Les maisons d'édition commencent probablement à se rendre compte que le succès des mangas ne sera pas éternel, sans doute parce que la première génération de ses lecteurs a grandi. C'est, je crois, l'une des raisons qui a permis au roman graphique de se faire une place. Mais ne nous leurrons pas: l'ex-fan de mangas, même adulte, ne deviendra certainement pas automatiquement un lecteur inconditionnel de romans graphiques, et je doute que le boom des mangas soit remplacé par un boom des romans graphiques! 
JH : L'Allemagne a vu émerger des auteurs de « comics » indépendants qui s'inspirent - mais sans pour autant les copier ! - à la fois des mangas, mais aussi des écoles de BD classiques. Et puis il y a des mangas allemands, très proches de leurs modèles japonais. Mais c'est un univers très différent de celui des jeunes auteurs et dessinateurs de romans graphiques.

PM : Il n'y a malheureusement pas d'équivalent allemand pour le terme "graphic novel ». J'avais essayé de proposer « récit graphique » (grafische Erzählung), mais la langue allemande aime trop les américanismes. J'imagine que les gens pensent que ça sonne mieux, que c'est plus intéressant. À mon avis, c'est bien regrettable. Avec les romans graphiques, les éditeurs de $\mathrm{BD}$ allemands ont réussi à conquérir les librairies. Avant, les BD se vendaient presque exclusivement dans des boutiques spécialisées et les chiffres de vente étaient par conséquent très modestes. Les librairies offrent des possibilités bien supérieures pour ce qui concerne la vente. La première édition de Giftest par exemple déjà épuisée - après six mois seulement en rayon! Dans une boutique spécialisée uniquement dans la BD, une telle chose aurait été impensable ! Le terme de roman graphique permet ainsi d'atteindre de nouveaux lecteurs, en passant par les librairies. Et pour l'instant, ça fonctionne très bien ! On peut donc dire en toute légitimité que le marché allemand de la BD a connu un véritable tournant, même si ce n'est pas seulement grâce au roman graphique. La BD fait enfin partie des pratiques culturelles des Allemands. Aujourd'hui, il va de soi que les grands quotidiens allemands aient leur rubrique $\mathrm{BD}$ et rendent compte des dernières parutions. C'était impensable il y a vingt ans!

Peut-on dire que l'ère caractérisée par une influence des auteurs américains ou francophones est révolue en Allemagne?

BY : Mais non, ces influences existent encore, il serait regrettable de rétrécir ainsi l'horizon! Il faut voir les influences comme des enrichissements, et tant mieux si la réciprocité se développe!

IK : Ce ne sont certainement plus les seules influences que l'on trouve aujourd'hui dans la BD germanophone.

PM : Oui, ces temps sont révolus, heureusement ! Comment expliquer autrement que de plus en plus d'albums allemands sont traduits en français ? Le nouveau paysage de la $\mathrm{BD}$ de langue allemande est tout à fait particulier, et c'est ça qui intéresse les gens en France (et ailleurs aussi !). Des styles graphiques nouveaux, des thèmes que l'on n'abordait pas dans un " comic " auparavant. La BD allemande possède aujourd'hui une originalité propre, une originalité dont on n'aurait pas osé rêver il y a vingt ans !

L'humour est international, mais ses formes "nationales » ne franchissent pas très facilement les frontières. Nicolas Mahler, vous êtes un BDiste autrichien: comment expliquez-vous que vos albums soient également connus et appréciés en France ? Est-ce la même chose en Allemagne? Et plus globalement: quelles différences et/ou ressemblances entre le monde de la BD en Allemagne et en Autriche?

$\mathrm{NM}$ : En fait, mes travaux ne sont que partiellement connus en France. Mes cartoons vraiment humoristiques n'ont pas été traduits. Ce sont des "comic» courts qui ne fonctionnent pas bien dans le contexte français. Il est plus facile de transposer dans d'autres langues les narrations plus longues, entre autres parce que j'essaie d'y éviter tout rapport trop direct avec l'actualité concrète. Mes personnages sont en réalité plutôt des archétypes que l'on comprend partout. Ce n'était pas une intention de ma 
part, c'était un peu le fruit du hasard. Ce sont plutôt des récits de vie. C'est probablement dans ma manière de raconter ces histoires, de les raconter avec un humour un peu particulier et assez inhabituel en France, que l'on peut trouver la raison pour laquelle mes BD y suscitent cet intérêt. Pour la seconde partie de la question : il est toujours difficile de comparer deux milieux de la BD... Je dirais que le milieu allemand est plus actif, avec un réseau plus efficace. En Autriche, nous nous battons plutôt en solitaires...

Comment voyez-vous l'avenir de la BD, ou du roman graphique, en Allemagne?

$\mathrm{JH}$ : En tant que membre de l'équipe de la maison d'édition REPRODUKT je suis très optimiste. Je crois que l'évolution positive constatée au cours de ces dernières années continuera : la critique littéraire s'ouvre à la $\mathrm{BD}$, laquelle est toujours davantage prise au sérieux par les libraires et les lecteurs.

On peut dire que la lecture des BD deviendra bientôt une pratique culturelle courante en Allemagne.

IK : En tant que dessinatrice de BD qui se réjouit du succès du roman graphique en Allemagne, je ne peux qu'espérer que l'intérêt des maisons d'édition, du marché du livre et de la presse ne faiblira pas...

$\mathrm{PM}$ : Je ne peux vous répondre qu'une chose: voyez le succès de l'exposition d'Erlangen ${ }^{2}$ !

NM: Il y a maintenant, à mon initiative, des distributeurs de BD dans le Museumsquartier de Vienne, un lieu très fréquenté en plein centre-ville. Leur avantage: les gens n'ont plus besoin d'aller dans une boutique spécialisée pour acheter une BD. Les clients sont tout simplement ceux qui visitent les musées ou les restaurants du Museumsquartier. C'est une clientèle de passage idéale! Et c'est ainsi que nous espérons gagner de nouveaux lecteurs, c'est ça l'objectif...

Jutta Harms, pendant longtemps, «BD » était généralement synonyme d'« humour ». Or beaucoup de romans graphiques actuellement sur le marché traitent de sujets plutôt sombres. Est-ce seulement dû au hasard?

$\mathrm{JH}$ : C'est une évolution qui signifie que de plus en plus d'auteurs utilisent ce médium pour raconter leurs histoires sous forme de BD. Dans la littérature et au cinéma, on débat et réfléchit aussi sur des questions importantes pour notre société. Peut-être est-ce tout simplement le signe que la BD est devenue adulte...

\section{Notes de présentation :}

\section{Dessinateurs :}

4 Isabel Kreitz, née en 1967 à Hambourg, a étudié les arts graphiques dans sa ville natale et à New York. En 1997, elle reçoit au Festival de la bande dessinée de Hambourg le titre de Meilleure dessinatrice allemande de B.D. pour l'adaptation du roman d'Uwe Timm : Die Entdeckung der Currywurst (Carlsen Verlag, 1996). Dix ans plus tard, elle adapte également Der 35. Mai d'Erich Kästner (C. Dressler-Verlag, 2006), puis réalise en 2008 l'un des plus grands succès de librairie en Allemagne dans le domaine des romans graphiques avec Die Sache mit Sorge (Carlsen), pour lequel elle obtient la même année le Prix Max-und-Moritz au Salon international de la bande dessinée d'Erlangen. Cet ouvrage est paru en France sous le titre L'espion de Staline (traduction: Paul Derouet) 
aux Éditions Casterman. Le nouveau roman graphique d'Isabel Kreitz (Haarmann, d'après un scénario de Peer Meter) paraittra en Allemagne en octobre 2010 chez Carlsen. Casterman en publiera la traduction française en avril 2011.

dessin satirique en Autriche. La notoriété de cet auteur très publié (près de 30 livres parus) a rapidement dépassé les frontières de son pays, grâce à la publication de ses dessins et de ses strips par de nombreux supports de presse, en Allemagne (FAZ) comme en Suisse (Strapazin), et grâce à la parution de plusieurs de ses livres en France, publiés par « L'Association ». Militant du $9^{e}$ art dans son pays, il est le cofondateur du « Kabinett für Wort und Bild» (2003) au Museumsquartier de Vienne (bel espace culturel, en plein cœur de la capitale), qui propose notamment des comics à la vente dans des distributeurs automatiques. Ses productions sur papier et les dessins animés qui en sont tirés, mettent en scène des personnages (Flaschko, $\mathrm{M}^{\mathrm{me}}$ Goldgruber) au graphisme minimaliste mais très évocateur. Mahler a reçu en juin dernier le Prix du meilleur auteur de langue allemande au XIV ${ }^{e}$ Salon international de la bande dessinée d'Erlangen. À cette occasion, une grande exposition présentait les différentes facettes de son travail. Si vous voulez en apprendre davantage, consultez son site internet www.mahlermuseum.at ou allez sur le site de la librairie viennoise Pictopia (www.pictopia.at).

7 Barbara Yelin est née en 1977 à Munich et s'est installée à Berlin après des études d'arts graphiques (illustration) à Hambourg, où elle a été l'élève de l'une des figures de proue du dessin en Allemagne, Anke Feuchtenberger (www.feuchtenbergerowa.de). C'est en France (Éditions de l'An 2) que paraissent ses deux premiers albums, publiés par l'excellent découvreur de talents qu'est Thierry Groensteen, ancien directeur du Centre national de la bande dessinée et de l'image d'Angoulême. Bien que très peu connue du grand public allemand (son travail n'était publié que dans des ouvrages collectifs comme l'album Pomme d'amour, paru en 2008, ou le magazine berlinois Spring), B. Yelin est désignée à la Foire du Livre de Francfort comme nouveau talent (« Newcomer ») de l'année 2008, dans la catégorie B.D. (« Comic-Preis Sondermann »).

Grâce au succès de son dernier ouvrage, Gift, roman graphique impressionnant qui retrace la vie de Gesche Gottfried (Reprodukt, mars 2010) à partir d'un scénario de Peer Meter (paru en français sous le titre L'Empoisonneuse chez Actes Sud/L'An 2, avril 2010), Barbara Yelin est désormais l'une des étoiles montantes de la nouvelle génération. Son site www.barbarayelin.de donne un aperçu de son travail en tant qu'illustratrice et comme membre $\mathrm{du}$ collectif d'artistes berlinois «Bilderbureau » (www.bilderbureau.de).

\section{Scénariste :}

9 Peer Meter, scénariste, est un écrivain allemand né en 1956, qui vit à Worpswede (Basse-Saxe). En tant que scénariste de bandes dessinées et de romans graphiques, il fournit à de nombreux jeunes dessinateurs des sujets caractérisés par leur complexité et leur originalité. Ses derniers ouvrages (Gift avec Barbara Yelin, Die Sache mit Sorge avec Isabel Kreitz, Vasmers Bruder avec David von Bassewitz) qui donnent à voir des 
univers sombres et des personnages hors normes ont été accueillis avec enthousiasme par le public et la critique.

10 En juin 2010, le XIV Salon international de la bande dessinée d'Erlangen lui a consacré une exposition passionnante intitulée "Sechs Zeichner, ein Autor ", qui présentait le travail de six dessinateurs (Barbara Yelin, Isabel Kreitz, David von Bassewitz, Gerda Raidt, Nicola Maier-Reimer et Julia Briemle) à partir de scenarii de Peer Meter. Le site de ce dernier (www.peermeter.de) fourmille d'informations sur l'actualité de la bande dessinée en Allemagne.

\section{Monde de l'édition :}

11 Jutta Harms, éditrice, travaille pour la jeune maison d'édition berlinoise REPRODUKT, qui a été fondée en 1991 et propose environ 25 nouveaux titres par an. Très soucieuse de qualité, REPRODUKT publie des albums originaux d'auteurs germanophones mais également des traductions d'auteurs étrangers, notamment francophones. Elle participe activement au site internet www.graphic-novel.info commun à cinq maisons d'édition spécialisées dans la bande dessinée germanophone (une grande première et une belle réussite!), qui regroupe les éditeurs Carlsen Comics, Reprodukt, Edition 52, Avant-Verlag et Edition Moderne ( $\mathrm{CH})$. 